\title{
A Synchronization Scheme Using Self-Pulsating Laser Diodes in Optical Chaotic Communication
}

\author{
Cheng Juang, T. M. Hwang, J. Juang, and Wen-Wei Lin
}

\begin{abstract}
A synchronization scheme using self-pulsating laser diodes in optical chaotic communication is proposed. Optical chaotic light can be obtained by injecting a sinusoidal electronic signal into a self-pulsating laser diode. To synchronize between two identical chaotic systems with different initial conditions, a drive and response system model is constructed according to Pecora and Carroll's theory. Synchronization can be achieved for optical simplex and duplex transmissions provided that the conditional Lyapunov exponents for the drive and response systems are all negative. This approach offers a key step toward realizing optical chaotic modulation and demodulation.
\end{abstract}

Index Terms-Conditional Lyapunov exponents, optical chaotic communication, self-pulsating laser diodes, synchronization.

\section{INTRODUCTION}

C ARRIER synchronization (lock-in) between two chaotic systems is the key to establishing chaotic communication channels. The property of chaotic systems is that closely correlated initial conditions have trajectories which quickly become uncorrelated. This property would seem to defy synchronization. However, Pecora and Carroll [1], [2] have shown that two chaotic systems can be synchronized provided that the systems meet the following criteria. First, the first chaotic (drive) system transmits some of its state variable to the second (response) system. This forces the state variables of the response system to synchronize with the other state variables not passed to the response system. Second, a necessary and sufficient condition for the synchronization is that all the conditional Lyapunov exponents (CLE's) associated with the variational equation be negative. The ability to design synchronizing systems has opened opportunities for applications of chaos to private communications [3].

In optical chaotic communications which have the potential for high-speed communications, class B lasers (master-slave model) have been used to achieve the synchronization and signal transmission [4]. In this work, a drive-response model according to Pecora and Carroll's theory is used [1], [2]. Three issues have to be addressed in order to apply Pecora and Carroll's theory: 1) masking the electronic signal with optical chaotic light;, 2)

Manuscript received August 11, 1999; revised November 24, 1999. This work was supported by the National Science Council R.O.C., under Contract 89-2215-E159-001, Contract 89-2115-M009-004, and Contract 89-2115-M007-0023.

C. Juang is with the Electronics Department, Ming Hsin College, Hsinfeng, Hsinchu, Taiwan, R.O.C.

T. M. Hwang and W.-W. Lin are with the Department of Mathematics, National Tsing Hua University, Hsinchu 300, Taiwan, R.O.C.

J. Juang is with the Department of Applied Mathematics, National Chiao

Tung University, Hsinchu, Taiwan, R.O.C.

Publisher Item Identifier S 0018-9197(00)01817-0. transmitting, receiving, and coupling at least one state variable between the drive and response systems; and 3) obtaining all the negative CLE's for the drive and response systems at one particular drive electronic signal. It has been known that, by injecting a sinusoidal electronic signal to laser diodes [5], [6], and in particular, self-pulsating laser diodes [7], [8], chaotic light can be generated in certain conditions. In this way, self-pulsating laser diodes can be used as chaotic masking devices. In addition, a reasonable (probably the only one) choice for this state variable to be transmitted between the drive and response systems is photon density. Note that, if all the state variables are passed to the response system, the total system becomes trivial. Photon density could then be received, processed, and coupled into the response system in the form of current injection. Finally, to meet condition 3) above, the CLE's are calculated at this particular electronic drive.

A synchronization scheme using self-pulsating laser diodes in optical chaotic communications is proposed in this paper. Section II formulates a possible drive-response system model in optical chaotic simplex and duplex transmission according to Pecora and Carroll's theory. Section III plots the bifurcation diagram from the Poincaré map and computes the Lyapunov exponents for self-pulsating laser diodes under sinusoidal electronic drive. Synchronization of the simplex and duplex transmissions is described in Sections IV and V, respectively. Effects of coupling coefficients on the CLE's and synchronization are studied.

\section{FORMULATION}

\section{A. Drive-Response System}

Considering a drive-response system using self-pulsating laser diodes as chaotic masking devices, a drive system described by a three-dimensional (3-D) rate equation is given by

$$
\begin{aligned}
\frac{d N_{1}}{d t} & =F_{1}\left(N_{1}, N_{2}, S\right)+a+b \sin 2 \pi f t+\delta(\hat{S}-S) \\
\frac{d N_{2}}{d t} & =F_{2}\left(N_{1}, N_{2}, S\right) \\
\frac{d S}{d t} & =F_{3}\left(N_{1}, N_{2}, S\right)
\end{aligned}
$$

and the appropriate response system ( ${ }^{\wedge}$ ) is given by

$$
\begin{aligned}
\frac{d \hat{N}_{1}}{d t} & =\hat{F}_{1}\left(\hat{N}_{1}, \hat{N}_{2}, \hat{S}\right)+\hat{a}+\hat{b} \sin 2 \pi \hat{f} t+\hat{\delta}(S-\hat{S}) \\
\frac{d \hat{N}_{2}}{d t} & =\hat{F}_{2}\left(\hat{N}_{1}, \hat{N}_{2}, \hat{S}\right) \\
\frac{d \hat{S}}{d t} & =\hat{F}_{3}\left(\hat{N}_{1}, \hat{N}_{2}, \hat{S}\right)
\end{aligned}
$$


TABLE I

PARAMETERS USED FOR SimUlation OF Self-PUlsating Laser Diodes

\begin{tabular}{lll}
\hline$k_{1}$ & $3.08 \times 10^{-12}$ & $\mathrm{~m}^{3} / S$ \\
$k_{2}$ & $1.232 \times 10^{-11}$ & $\mathrm{~m}^{3} / S$ \\
$\xi_{1}$ & 0.2034 & - \\
$\xi_{2}$ & 0.1449 & - \\
$N_{g 1}$ & $1.4 \times 10^{24}$ & $\mathrm{~m}^{-3}$ \\
$N_{g 2}$ & $1.6 \times 10^{24}$ & $\mathrm{~m}^{-3}$ \\
$V_{1}$ & 72 & $\mu m^{3}$ \\
$V_{2}$ & 102.96 & $\mu m^{3}$ \\
$T_{12}$ & 2.65 & $n s$ \\
$T_{21}$ & 4.452 & $n s$ \\
$G_{t h}$ & $3.91 \times 10^{11}$ & $S^{-1}$ \\
$C$ & $1.573 \times 10^{-23}$ & $\mu m^{-3}$ \\
$\tau_{s}$ & 3 & $n s$ \\
\hline \hline
\end{tabular}

where $S$ is the photon density, $N_{1}$ is the electron density in the active region, $N_{2}$ is the electron density in the saturable absorption region, $I=a+b \sin 2 \pi f t+\delta(\hat{S}-S)$ is the injection current, and $\delta$ is the coupling coefficient. Note that $I$ is normalized using a factor of $e V_{1}$, where $V_{1}$ is the active layer volume. The nonlinear functions $F_{1}, F_{2}$, and $F_{3}$, which describe the self-pulsating laser diodes, are written as [9]

$$
\begin{aligned}
F_{1}= & -\frac{k_{1} \xi_{1}}{V_{1}}\left(N_{1}-N_{g 1}\right) S-\frac{N_{1}}{\tau_{s}}-\frac{N_{1}-N_{2}}{T_{12}} \\
F_{2}= & -\frac{k_{2} \xi_{2}}{V_{2}}\left(N_{2}-N_{g 2}\right) S-\frac{N_{2}}{\tau_{s}}-\frac{N_{2}-N_{1}}{T_{21}} \\
F_{3}= & {\left[k_{1} \xi_{1}\left(N_{1}-N_{g 1}\right)+k_{2} \xi_{2}\left(N_{2}-N_{g 2}\right)-G_{\mathrm{th}}\right] S } \\
& +C \frac{N_{1} V_{1}}{\tau_{s}}
\end{aligned}
$$

where

$\tau_{s} \quad$ carrier lifetime;

$\xi \quad$ confinement factor;

$G_{\text {th }}$ threshold gain level;

$T$ carrier time diffusion constant between the two layers;

$k \quad$ linear approximation constant for the gain curve;

$N_{g} \quad$ transparent level of electron density;

$C$ coupling ratio between the spontaneous field and the lasing mode.

The subscripts 1 and 2 describe terms in the active and absorption layers, respectively.

Table I lists all the parameters of the self-pulsating laser diode obtained from [9] used in the simulation. Increasing the bias current yields a dramatic change in output light at $19.8 \mathrm{~mA}$, which corresponds to the threshold current of the laser. When the layer is above threshold, the self-pulsating frequency $f_{0}$ increases due

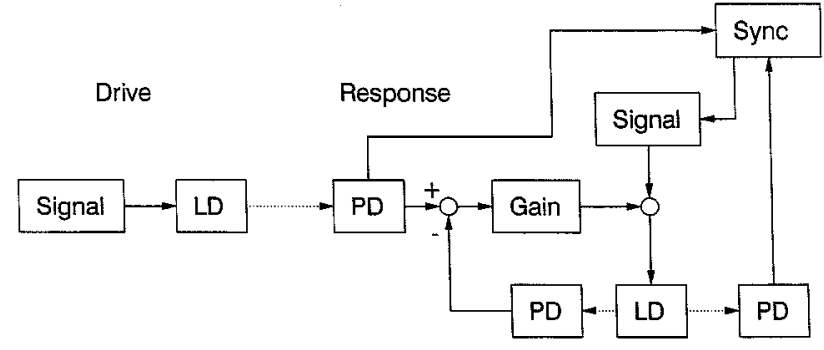

Fig. 1. An optical chaotic simplex transmission block diagram.

to the increase in bias current. When $a=30 \mathrm{~mA}$ is injected, the corresponding $f_{0}$ is $2.28 \mathrm{GHz}$. This is used in the calculations throughout.

\section{B. Simplex and Duplex Transmission}

Equations (1) and (2) are general forms expressing two-way (duplex) transmission between drive and response systems. If $\delta$ is zero, the system becomes one-way (simplex) transmission. Fig. 1 illustrates a possible transmission block diagram for a simplex system. The drive system sends $S(t)$ to the response system and then subtracts $\hat{S}(t)$, which is from the monitor photodetector of the identical self-pulsating laser diode in the response system. The tunable gain stage corresponds to the coupling coefficient $\hat{\delta}$. Mixed with the signal current again, the total injection current becomes $\hat{I}=\hat{a}+\hat{b} \sin 2 \pi \hat{f} t+\hat{\delta}(S-\hat{S})$. Thus, chaotic simplex transmission system can be implemented.

\section{Chaotic Masking Using Self-Pulsating LaSer DIODES}

By injecting a sinusoidal electronic signal into a self-pulsating laser diode $(I=a+b \sin 2 \pi f t)$, optical chaotic light can be obtained. In order to characterize the asymptotic behavior of the drive system, numerical computations on Poincaré maps and Lyapunov exponents are carried out.

\section{A. Poincaré Map}

In the 3-D phase diagram $\left(N_{1}, N_{2}\right.$, and $\left.S\right)$ of the rate equations, let $\Sigma$ be a two-dimensional 2-D hyperplane through a point $(0,0.298,0)$ with the normal direction $[0,1,0]$. Consider the Poincaré map $\mathcal{P}: \Sigma \longrightarrow \Sigma$ defined by $\mathcal{P}(x):=$ $\phi_{t+t_{x}}\left(x, t_{o}\right)$, where $\mathcal{P}(x)$ denotes the first return point at $\Sigma$ of the trajectory $\phi_{t+t_{x}}\left(x, t_{o}\right)$ in time $t_{x}$, which emanates from $x \in \Sigma$ starting at the initial time $t_{o}$. It is known that a finite set $\left\{x_{1}, \cdots, x_{k}\right\}$ of the trajectory intersecting a hyperplane $\Sigma$ results in a system with a period- $k$ solution. If the points $\mathcal{P}^{k}(x), k=1,2, \cdots$ densely fill out the closed curve, then the solution forms a quasi-two-periodic orbit. A Poincaré map program is written according to the pseudocode in [10].

Fig. 2 shows the bifurcation diagram of the Poincaré map with $S(t)$ versus $b$ for $f=0.3 f_{0}$. When $b \in(0,4)$ the system has a quasi-two-period attractor. When $b$ varies from 4 to 6 , the effects of quasi-periodicity routes to chaos are observed. The system has a period-3 window at $b \in(6,8.33)$ and period-doubling occurs when $b>8.33$. The phenomenon of the period-doubling routes to chaos occurs when $b \approx 8.35$. 


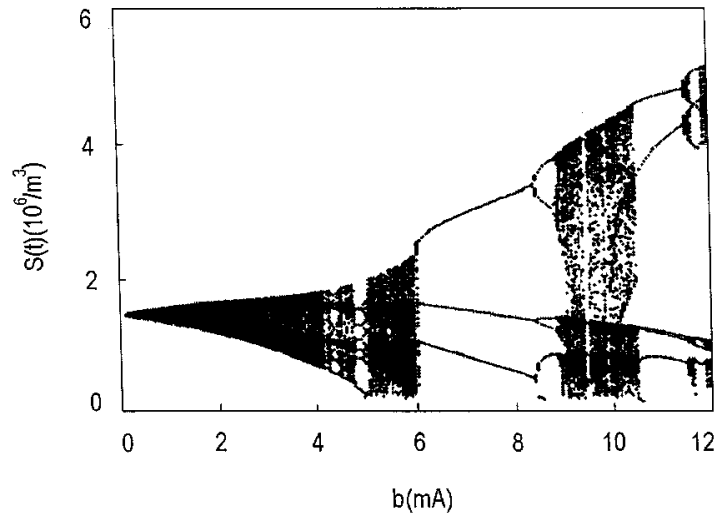

Fig. 2. Bifurcation diagram of a Poincaré map with $S(t)$ versus $b$ for $f=$ $0.3 f_{0}$, and $a=30 \mathrm{~mA}$.

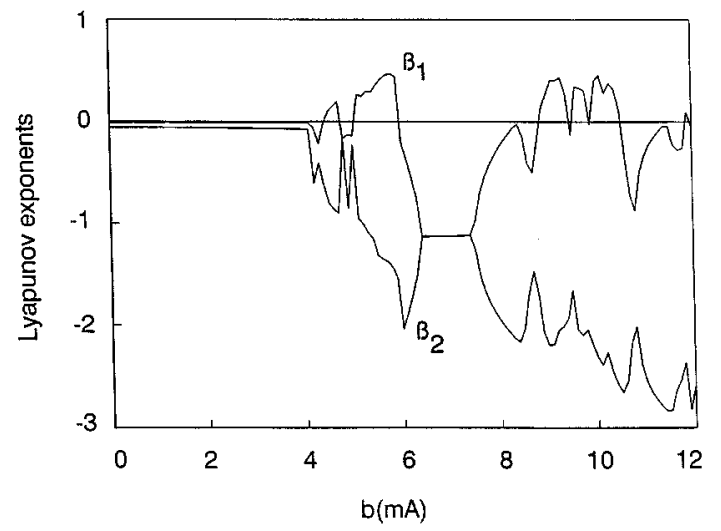

Fig. 3. The first two Lyapunov exponents of drive systems $\beta_{1}$ and $\beta_{2}$ versus $b$ for $f=0.3 f_{0}$ and $a=30 \mathrm{~mA}$.

\section{B. Lyapunov Exponents for Self-Pulsating Laser Diodes}

Lyapunov exponents are the generalization of the eigenvalues at an equilibrium point of characteristic multipliers. They can be used to determine the stability of quasi-periodic and chaotic behaviors, as well as that of equilibrium points and periodic solutions. Let $m_{1}(t), \cdots, m_{n}(t)$ be the eigenvalues of $\Phi_{t}\left(x_{0}\right)$, which is the transition matrix for $\Phi_{0}\left(x_{0}\right)=I_{n}$. The Lyapunov exponents of $x_{0}$ are defined by

$$
\beta_{i}=\lim _{t \rightarrow \infty} \frac{1}{t} \ln \left|m_{i}(t)\right|
$$

for $i=1, \cdots, n$, whenever the limits exist. A practical algorithm was developed according to the pseudocode in [10]. This algorithm requires more than $2 \times 10^{6}$ iteration steps to compute the Lyapunov exponents to the required degree of accuracy.

Fig. 3 shows the first two Lyapunov exponents $\beta_{1}$ and $\beta_{2}$ versus $b$. Note that the third Lyapunov exponents are all negative $(\leq-5)$. For $b \in(0,4), \beta_{1} \approx \beta_{2} \approx 0$, and $\beta_{3}<0$. This implies a nonchaotic quasi-two-period attractor which agrees with the results from the Poincaré map. Because at least one Lyapunov exponent of a chaotic system must be positive, chaotic behavior can be established in regions where one positive Lyapunov exponent is shown in the figure. The system also has a period-3 window for $b \in(6,8.33)$; therefore, in this region, there is no positive Lyapunov exponent.

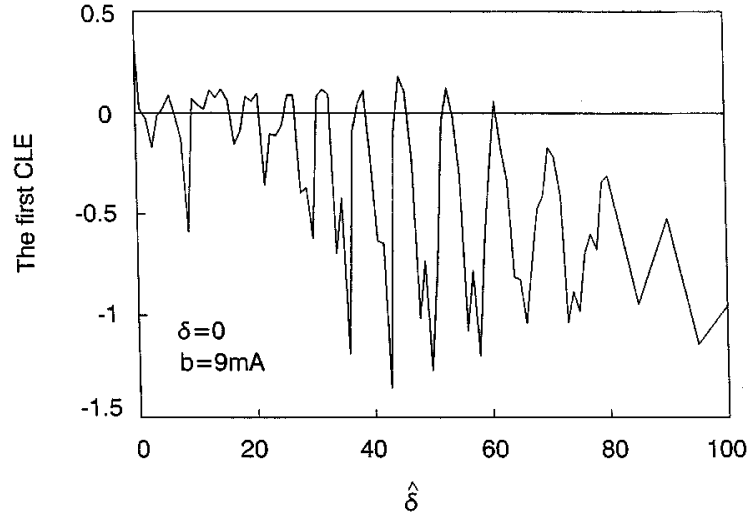

Fig. 4. The first conditional Lyapunov exponent of the drive-response system versus $\hat{\delta}$ for $\delta=0, f=0.3 f_{0}, b=9 \mathrm{~mA}$, and $a=30 \mathrm{~mA}$

\section{SYNCHRONIZATION OF SIMPLEX TRANSMISSION}

When the drive system sends a chaotic state variable $S(t)\left(f=0.3 f_{0}, b=9 \mathrm{~mA}\right.$, and $\left.\beta_{1}>0\right)$ to the same response system with slightly different initial conditions $\left(N_{1}(0)=N_{2}(0)=S(0)=10^{-6}\right)$, synchronization between the two state variables $S(t)$ and $\hat{S}(t)$ can be achieved.

\section{A. Conditional Lyapunov Exponents}

According to Pecora and Carroll's theory, synchronization can only be achieved for all negative CLE's. The CLE's are found by calculating the Lyapunov exponents for the entire system and comparing these to the Lyapunov exponents of the drive system. The remaining Lyapunov exponents are the CLE's. A simple method is to construct a difference system. Let $E_{N_{1}}=N_{1}-\hat{N}_{1}, E_{N_{2}}=N_{2}-\hat{N}_{2}$ and $E_{S}=S-\hat{S}$. The difference system becomes

$$
\frac{d E(t)}{d t}=A E(t) .
$$

The real part of the eigenvalues $\left(\lambda_{1}, \lambda_{2}\right.$, and $\left.\lambda_{3}\right)$ of $A^{-1}$ are the CLE's by definition. If all the CLE's are negative, $\lim _{t \rightarrow \infty} E(t)=0$ and the two systems will be synchronized. If there is a positive CLE, the difference system will grow further apart as $t \rightarrow \infty$.

Fig. 4 shows the first CLE of (5) against $\hat{\delta}$ when $\delta=0, f=$ $0.3 f_{0}$, and $b=9 \mathrm{~mA}$. Note that the second and third Lyapunov exponents are all negative. For $\hat{\delta} \leq 1.5$, there is at least one positive Lyapunov exponent $\left(\lambda_{1}>0>\lambda_{2}>\lambda_{3}\right)$. This implies that the difference system will grow further apart as $t \rightarrow \infty$. For $\hat{\delta} \geq 63$, there is no positive Lyapunov exponent $\left(0>\lambda_{1}>\right.$ $\lambda_{2}>\lambda_{3}$ ). This implied synchronization. In between, there is a mixed region where synchronization and nonsynchronization can both occur.

\section{B. Synchronization}

Synchronization can also be verified by directly solving (1) and (2). Fig. 5 shows $\hat{S}(t)$ versus $S(t)$ for weak coupling when $\hat{\delta}=1, \delta=0, f=0.3 f_{0}$, and $b=9 \mathrm{~mA}$. Drive and response systems with closely correlated initial conditions have trajectories which quickly become uncorrelated. In this case, there is a positive CLE. However, if the gain stage of the response system 


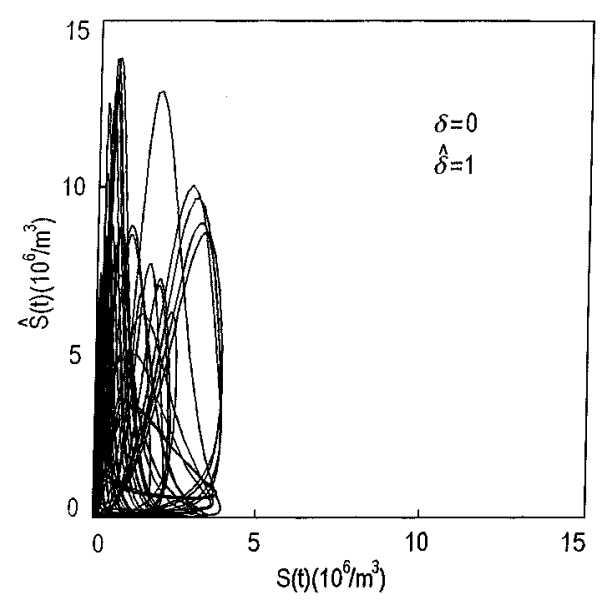

Fig. 5. $\quad \hat{S}(t)$ versus $S(t)$ for $\hat{\delta}=1, \delta=0, f=0.3 f_{0}$, and $b=9 \mathrm{~mA}$ in the simplex drive-response system.

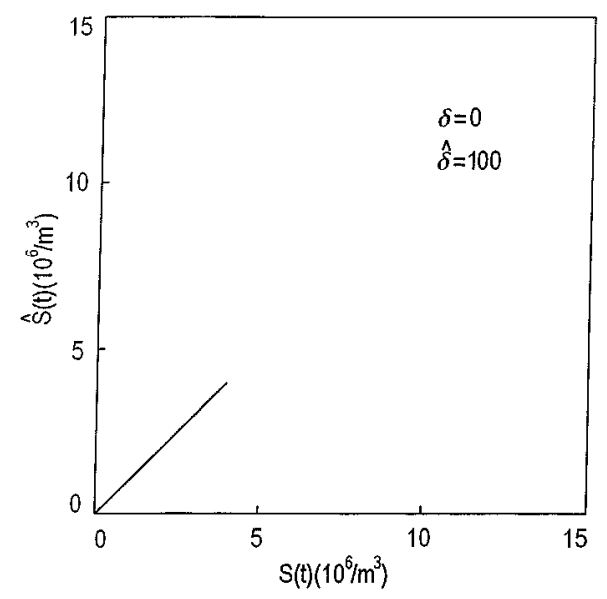

Fig. 6. $\hat{S}(t)$ versus $S(t)$ for $\hat{\delta}=100, \delta=0, f=0.3 f_{0}$, and $b=9 \mathrm{~mA}$ in the simplex drive-response system.

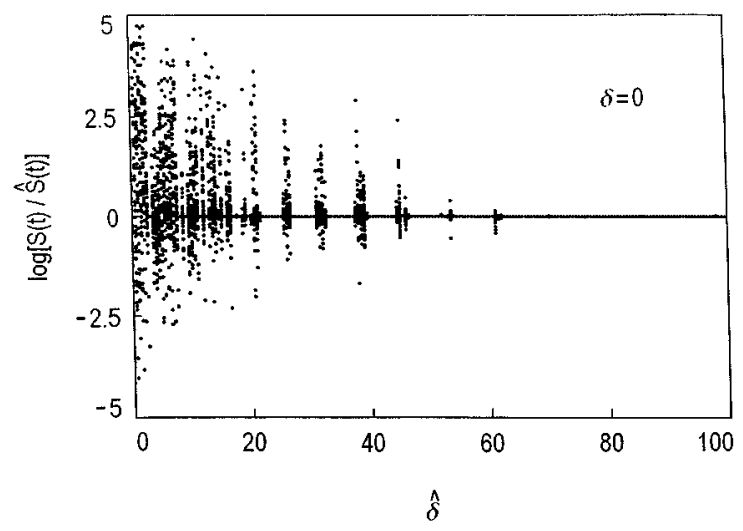

Fig. 7. $\log (\hat{S}(t) / S(t))$ against $\hat{\delta}$ for $\delta=0, f=0.3 f_{0}$, and $b=9 \mathrm{~mA}$ in the simplex drive-response system.

is tuned to a strong coupling $(\hat{\delta}=100)$, synchronization occurs as shown in Fig. 6. In this case, all CLE's are negative.

Fig. 7 plots $\log (\hat{S}(t) / S(t))$ against $\hat{\delta}$ for $\delta=0, f=0.3 f_{0}$, and $b=9 \mathrm{~mA}$. For $\hat{\delta} \geq 63, \log (\hat{S}(t) / S(t))=0$ implies that the drive and response systems are synchronized. When the value of $\log (\hat{S}(t) / S(t))$ varies, the two systems are not synchronized. In between 63 and 1.5, synchronization and nonsynchronization can occur. This result matches well with the CLE calculations.

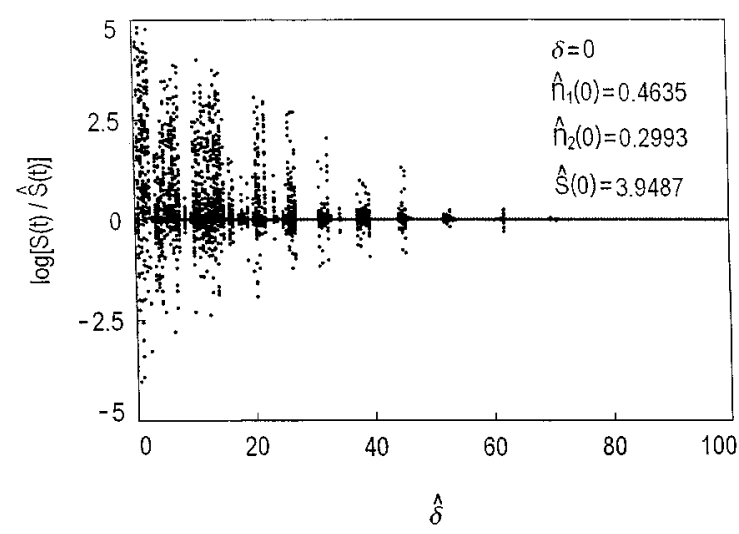

Fig. 8. $\log (\hat{S}(t) / S(t))$ against $\hat{\delta}$ for $\delta=0, f=0.3 f_{0}$, and $b=9 \mathrm{~mA}$ in the simplex drive-response system. The initial conditions of the response system have been changed to $\left(N_{1}(0)=0.4635, N_{2}(0)=0.2993\right.$, and $S(0)=$ 3.9487).

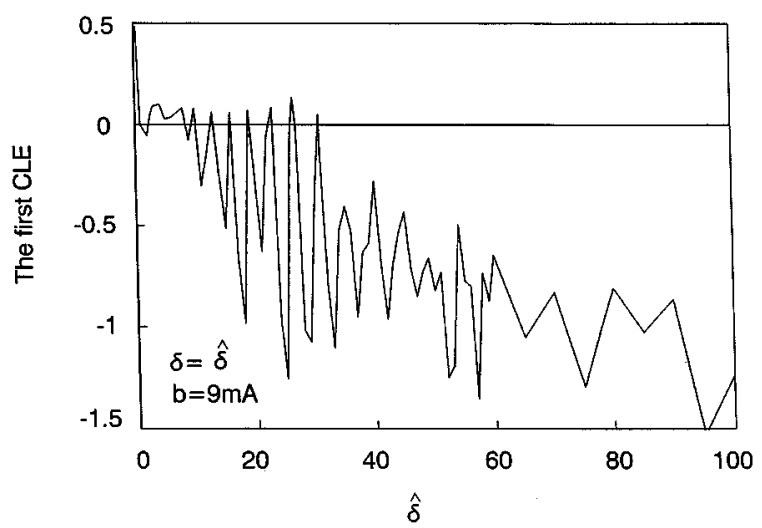

Fig. 9. The first conditional Lyapunov exponent of the drive-response system versus $\hat{\delta}$ for $\hat{\delta}=\delta, f=0.3 f_{0}, b=9 \mathrm{~mA}$, and $a=30 \mathrm{~mA}$.

With negative CLE's, all trajectories in the two systems approach each other asymptotically regardless of initial conditions provided that they are in the basin of attraction. To show that synchronization can be achieved under the worst-case initial condition (in a practical sense), Fig. 8 repeats Fig. 7 except that the initial conditions of the response system have been changed to $\left(N_{1}(0)=0.4635, N_{2}(0)=0.2993, S(0)=3.9487\right)$, where $S(0)$ is the maximum value for any $t$ of the response system without coupling. This result suggests that the drive and response systems have identical chaotic behaviors independent of initial conditions.

\section{SyNCHRONIZATION OF DUPLEX TRANSMISSION}

The drive system sends a chaotic state variable $S(t)$ to the response system. In return, the response system sends its chaotic state variable $\hat{S}(t)$ to the drive system. Thus, duplex (two-way) transmission can be established. Due to the exchange of state variables between the drive and response systems, it is expected that synchronization can be achieved more easily than in simplex transmission.

\section{A. Synchronization}

Fig. 9 shows the first CLE against $\hat{\delta}$ when $\hat{\delta}=\delta, f=0.3 f_{0}$, and $b=9 \mathrm{~mA}$. For $\hat{\delta} \geq 32$ (compare with 63 in the simplex 


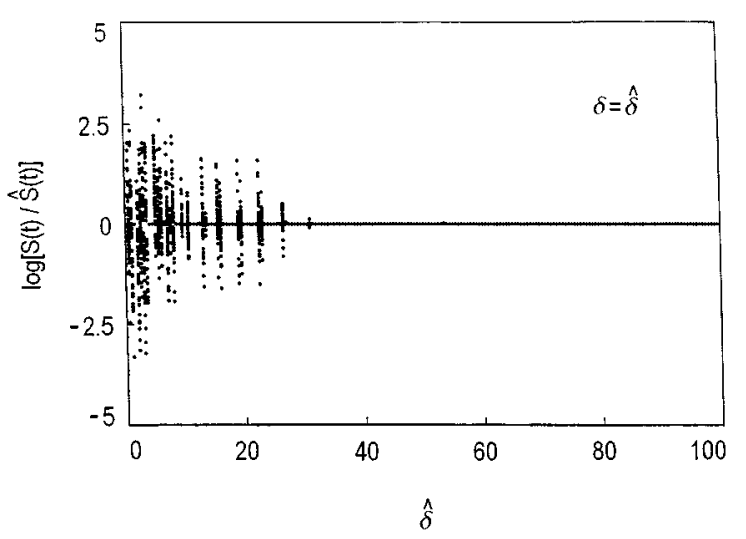

Fig. 10. $\log (\hat{S}(t) / S(t))$ versus $\hat{\delta}$ for $\hat{\delta}=\delta, f=0.3 f_{0}$, and $b=9 \mathrm{~mA}$ in the duplex drive-response system.

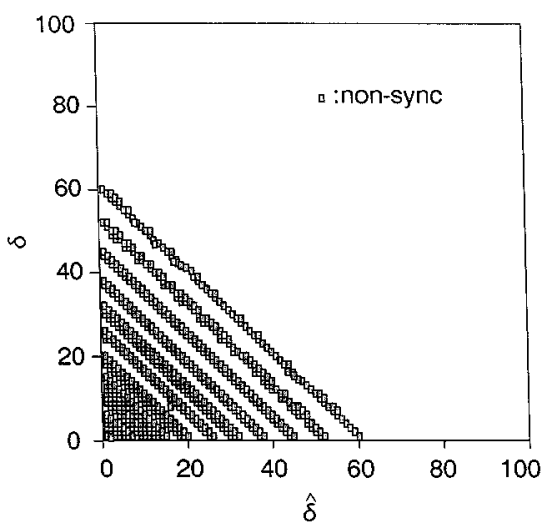

Fig. 11. Synchronization threshold of $\hat{\delta}$ and $\delta$ for $f=0.3 f_{0}$ and $b=9 \mathrm{~mA}$.

transmission), there is no positive CLE. The difference system will converge to zero and the drive and response systems will be synchronized.

Fig. 10 plots $\log (\hat{S}(t) / S(t))$ against $\hat{\delta}$ for $\hat{\delta}=\delta, f=0.3 f_{0}$, and $b=9 \mathrm{~mA} . \hat{S}(t)$ and $S(t)$ are directly solved from (1) and (2). For $\hat{\delta} \geq 32, \log (\hat{S}(t) / S(t))=0$ implies that the drive and response systems are synchronized.

\section{B. Synchronization Threshold}

Fig. 11 shows the synchronization threshold of $\hat{\delta}$ and $\delta$ for $f=0.3 f_{0}$ and $b=9 \mathrm{~mA}$. Dark points represent nonsynchronization regions. Thus, the synchronization thresholds can be approximated by a linear equation $(\hat{\delta}+\delta \geq 63)$. For all $\hat{\delta}$ and $\delta$ that satisfy the threshold equation, the corresponding drive and response systems will all be synchronized. This numerical finding has a simple explanation from (5) where $A$ can be approximated by the sum of the Jacobian of the uncoupled vector field and $(\hat{\delta}+\delta)$. Thus, when $\hat{\delta}=\delta$, the duplex system effectively has double the coupling strength of the simplex system and, hence, the ratio of the thresholds is around 2 .

\section{CONCLUSIONS}

Self-pulsating laser diodes can be used as chaotic masking devices to change sinusoidal electronic signals into optical chaotic light. By applying Pecora and Carroll's theory, one state variable (photon density) is transmitted between the drive and response systems (one-way for simplex and two-way for duplex). For strong coupling (all negative conditional Lyapunov exponents), synchronization can be achieved between the drive and response systems. Weakly coupled (one positive conditional Lyapunov exponent) drive and response systems quickly become uncorrelated.

\section{REFERENCES}

[1] L. M. Pecore and T. L. Carroll, "Synchronization in chaotic systems," Phys. Rev. Lett., vol. 64, pp. 821-824, 1990.

[2] - "Synchronization chaotic circuits," IEEE Trans. Circuit Syst., vol. 38, pp. 453-456, 1991.

[3] K. M. Cuomo, A. V. Oppenheim, and S. H. Strogatz, "Synchronization of Lorentz-based chaotic circuits with applications to communication," IEEE Trans. Circuit Syst. II, vol. 40, pp. 626-633, 1993.

[4] G. D. VanWiggeren and R. Roy, "Optical communication with chaotic waveforms," Phys. Rev. Lett., vol. 81, pp. 3547-3550, 1998

[5] S. Bennett, C. M. Snowden, and S. Iezekiel, "Nonlinear dynamics in directly modulated multiple-quantum-well laser diodes," IEEE J. Quantum Electron., vol. 33, pp. 2076-2083, 1997.

[6] E. Hemery, L. Chusseau, and J.-M. Lourtioz, "Dynamic behaviors of semiconductor lasers under strong sinusoidal current modulation: Modeling and experiments at $1.3 \mu \mathrm{m}$," IEEE J. Quantum Electron., vol. 26, pp. 633-641, 1990.

[7] H. G. Winful, Y. C. Chen, and J. M. Liu, "Frequency locking, quasiperiodicity, and chaos in modulated self-pulsating semiconductor lasers," Appl. Phy. Lett., vol. 48, pp. 616-618, 1986.

[8] C. Juang, M. R. Chen, and J. Juang, "Nonlinear dynamics of self-pulsating laser diodes under an external drive," Opt. Lett., vol. 24, pp. 1346-1348, 1999.

[9] M. Yamada, "A theoretical analysis of self-sustained pulsation phenomena in narrow-strip semiconductor lasers," IEEE J. Quantum Electron., vol. 29, pp. 1330-1336, 1993.

[10] T. S. Parker and L. O. Chua, Practical Numerical Algorithm for Chaotic Systems. Berlin, Germany: Springer-Verlag, 1989.

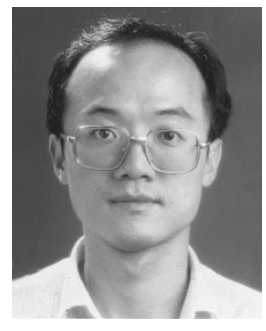

Cheng Juang received the B.S. degree in electronic engineering from Chiaotung University in 1981, the M.S. degree in electrical engineering from the University of Massachusetts, Amherst, in 1986, and the $\mathrm{Ph} . \mathrm{D}$. degree in electrical engineering from the University of Washington, Seattle, in 1990.

After graduation, he joined Optoelectronic's Laboratory, ITRI, in Hsinchu, Taiwan, as a Research Scientist. In 1993, he became a Faculty Member of the Electronics Department, Ming Hsin Institute, Taiwan. He is presently an Associate Professor and serves as the Department Chair. His research interests include nonlinear effects of laser diodes and quantum structures.

T. M. Hwang, photograph and biography not available at the time of publication.

J. Juang, photograph and biography not available at the time of publication.

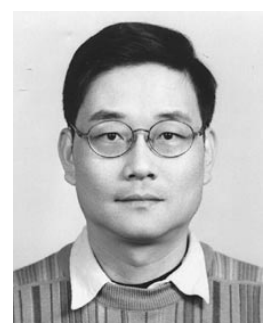

Wen-Wei Lin received the B.S. degree in applied mathematics from Chengchi University in 1976, the M.S. degree from Tsinghua University in 1978, and the Ph.D. degree from Bielefeld University, Germany, in 1985.

Currently, he is a Full Professor in the Department of Mathematics of Tsinghua University, Taiwan. His research interests are in the areas of computational dynamical systems, optimal control system, matrix computations, and numerical PDE. 